\title{
EXPERIMENTAL INVESTIGATIONS OF TRANSPORT PROPERTIES OF NATURAL THERMAL INSULATION MATERIALS
}

\author{
I. CONTÉ, X.F. PENG \& Y. ZHANG \\ Laboratory of Phase-Change and Interfacial Transport Phenomena, Department of Thermal Engineering, \\ Tsinghua University, Beijing, China.
}

\begin{abstract}
Experimental investigations were conducted to characterize the thermal insulation properties of different natural low-cost materials (coconut fibers and groundnut shell). A steady state method was used to measure the thermal conductivity by holding the sample between two concentric spheres with heat transferred mainly by conduction from the inner sphere (heat source) through the testing material. For comparison, the same experimental procedure was done for a standard and man-made insulation material, aluminum silicate fiber. The results showed that for all the materials, the thermal conductivity increases with temperature; and the rates of increase are very similar. Also, two experimental samples were made to measure the moisture transport of the two natural materials: one with groundnut shell (composed of void space and grain) and the other sample with coconut fibers (composed of void space and fibers). The percentage of water accumulation due to vapor absorption was calculated for each layer after a pre-set time of experiment $(1 \mathrm{~h}, 2 \mathrm{~h}, \ldots)$. A numerical investigation was performed to simulate the transfer process with a general and sound model, and to present the basic features of the transfer process.

Keywords: air and moisture transport, natural material, porous media, thermal conductivity, thermal insulation.
\end{abstract}

\section{INTRODUCTION}

Until recently, engineers and scientists in the thermal insulation industry attached importance only to the value of the thermal conductivity coefficient or the performance of the insulation materials. Important criteria such as the environment (raw renewable materials, ozone depletion, energy consumption, waste processing, etc.) and health (allergies, turbid respiration, cancers, etc.) were often given less importance, even completely ignored. Thermal insulation design involves the choice of insulations with an optimal combination of characteristics such as low effective conductivity, sufficient strength, and desired thermal diffusivity, as well as one which is likely to have a negligible change in these properties during the expected lifetime of the insulated system. The most important characteristics of thermal insulations are: low thermal conductivity, good mechanical properties (like shock-resistance), high resistance to water absorption, chemical resistance, durability, and low cost. When heat transfer occurs through a separating wall, because of the temperature difference water vapor diffusion also occurs due to the pressure difference across the wall. As the temperature within the insulation decreases, the moisture already existing in the insulation may condense. Thermal insulation carrying cold fluids suffers thermal degradation due to condensation of water. Thus, the value of the water vapor transfer coefficient of the insulation materials is as important as the value of the heat transfer coefficient. The thermal insulation requirements have continuously increased not only in terms of thermal properties but also with respect to indoor environment quality and the environmental impact. A further point of interest is the development of alternative materials, like sheep and cotton wool, and intelligent materials as transparent insulation, and dynamic materials with temperature dependent thermal conductivity properties [1]. Huge technological advances have been made in the search for ever-better thermal products; various fibers, loose fills, and cellular 
plastics have all captured a part of the market. Prefabricated panel building components have been developed for use in specific constructions. Nowadays, many natural materials (such as cork and coconut fiber) are used in thermal insulation owing to their relatively low thermal conductivity, low cost, and harmlessness to the environment and human health. These materials are commonly used for insulation of low temperature systems, such as cold stores and residential buildings. The thermal conductivity of pure and expanded cork is $0.04 \mathrm{~W} /(\mathrm{m} \mathrm{K})$ at $293 \mathrm{~K}$ with a density of $120 \mathrm{~kg} / \mathrm{m}^{3}$ [2]. Cork is still widely used, but has been replaced in most countries by polystyrene, polyurethane, foamed glass, glass wool, rock wool, and other artificial insulation materials. However, one main disadvantage of using most of the natural materials in thermal insulation is the low resistance to moisture absorption.

Many theoretical and experimental investigations have been performed on thermal conductivity, moisture absorption, and condensation in porous media, with the aim of applying the results to thermal insulation and other industrial applications. Kodah et al. [3] reported an experimental investigation to characterize the thermal insulation properties of different compounds of natural low cost reinforcements with polyurethane foam forming the matrix (foam only, foam and hay, foam and cane). They found that the use of cane as a natural fiber with foam improved the mechanical properties of the foam twofold in addition to improving the thermal insulation properties. Fan et al. [4] reported an experimental investigation of the temperature and water content distribution within porous fibrous battings sandwiched by an inner and outer layer of thin covering fabrics using a novel sweating, guarded hot plate. They found that most of the change in temperature occurred within the first half hour and moisture absorption by the hygroscopic fibers affected the temperature distribution. The water content accumulated with time, and higher water content was found in the outer region than in inner regions of the battings.

This article presents experimental investigations of thermal conductivity and water absorption of two natural low cost materials - coconut fiber and groundnut shell. A steady state method was used to measure the thermal conductivity of the natural materials by holding the sample between two concentric spheres with heat transferred mainly by conduction from the inner sphere (heat source) through the testing material. For the moisture absorption, the multilayered samples made with the testing materials were bound on their top by ice and the bottom surface was exposed to vapor with temperature close to $100^{\circ} \mathrm{C}$. The percentage of water accumulation due to vapor absorption was calculated for each layer. A numerical investigation was performed to simulate the transfer process with a general and sound model, and to present the basic features of the transfer process.

\subsection{Testing materials}

\section{EXPERIMENTAL MEASUREMENT OF THERMAL CONDUCTIVITY}

Coconut fibers and groundnut shells, both natural low-cost materials, are very abundant in nature. The coconut fiber used was obtained by keeping the fruit envelope in water for 2 or 3 days. The fiber obtained could reach $30 \mathrm{~cm}$ in length with an average diameter of $0.3 \mathrm{~mm}$ (Fig. 1). Coconut fiber is characterized by its high percentage of lignin (40\%), which accounts for its stiffness when used for carpet manufacturing. Coconut fiber may be used as panels, bulk, or rolls for wall insulation. Since it is flammable, it should be treated with boric salt to improve its resistance. Groundnut shell is the outer jacket of peanuts. Groundnut shell has low density and long durability, but must be protected from attack by insects. Very similar to wood, groundnut shell may be treated to different dimensions (Fig. 2); they can be treated industrially to get chips for making assemblies such as plywood. 


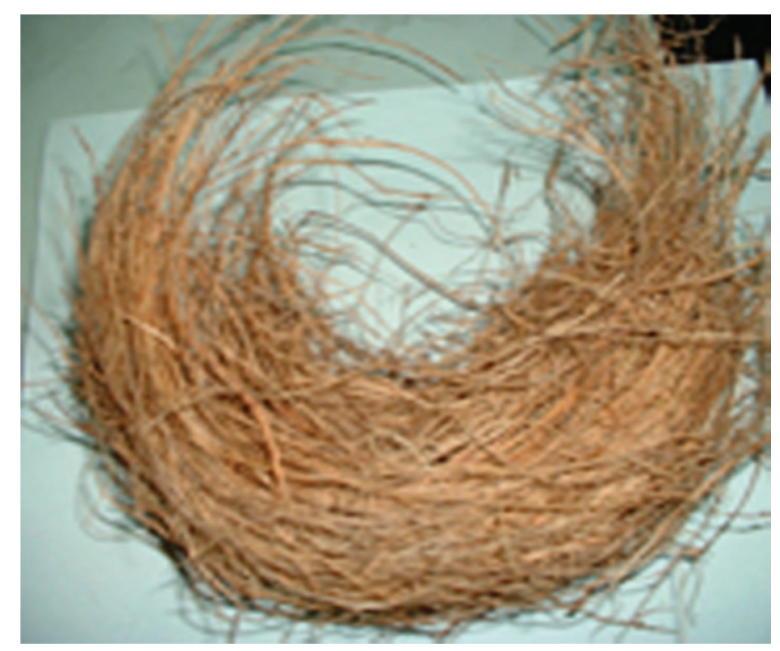

Figure 1: Coconut fibers.

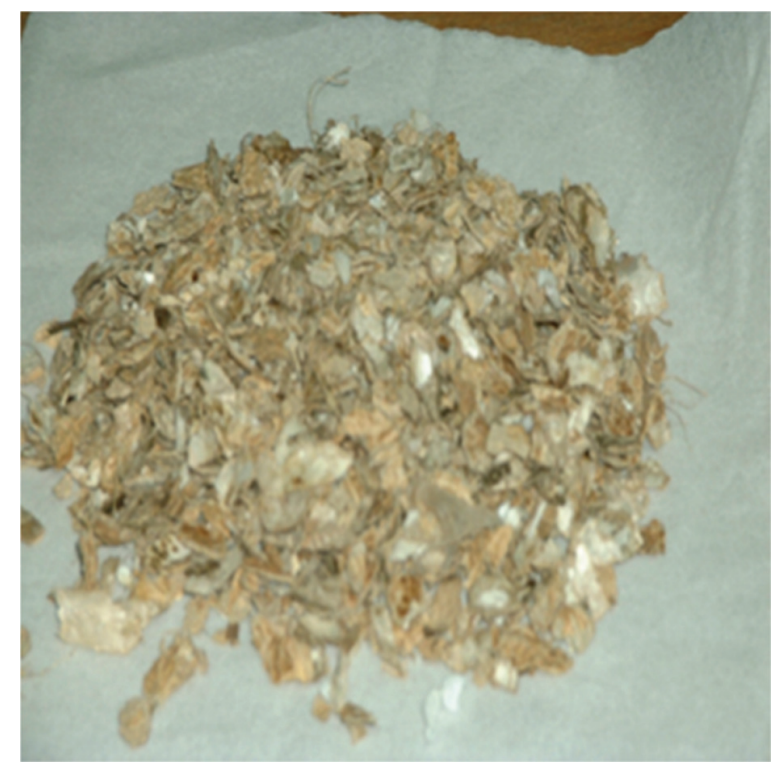

Figure 2: Groundnut shells.

The thermal conductivity of plywood varies with the timber species; however, an average value of $k=0.1154 \mathrm{~W} /\left(\mathrm{m}^{\circ} \mathrm{C}\right)[5]$ for softwood timbers is sufficiently accurate for determining the overall coefficient of heat transmission ( $U$ value) of a construction assembly. Thus, the decisive parameter for determining the thermal conductivity of any plywood should be that of the wood species. 


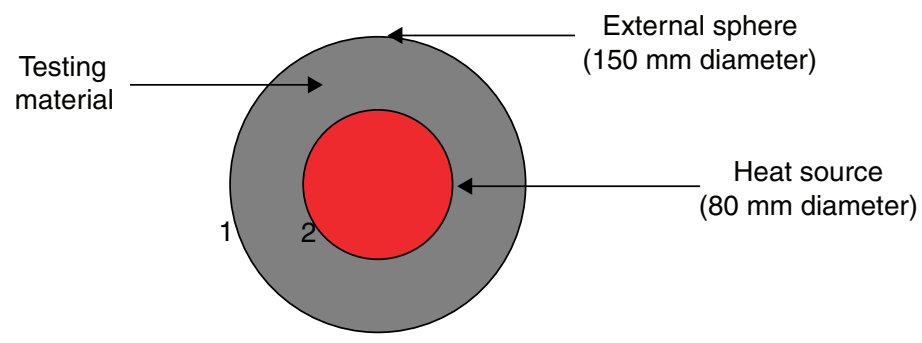

Figure 3: Schematic representation of the experimental module.

\subsection{Test apparatus}

A schematic representation of the experimental apparatus is shown in Fig. 3. The spherical enclosure measures $80 \mathrm{~mm}$ inside and $150 \mathrm{~mm}$ outside. The wall is made up of the material to be tested. Thermocouples are connected to the inner and outer copper spheres. A variable AC power supply and a watt meter were used to control and measure the power input. A Hewlett Packard 34401A multi-meter was used to record the temperature. The thermocouples were connected at points 1 and 2 (see Fig. 3).

\subsection{Experimental procedure}

The experimental procedure for the thermal conductivity measurement is as follows. First, the test materials are dried in a furnace at about $50^{\circ} \mathrm{C}$. The dried test materials are then put between the two concentric copper spheres. The test materials are carefully packed to achieve the greatest density and reduce the amount of air. The thermocouples are connected to the heat source (inner sphere) and the outer sphere. The entire setup is placed in a room with very negligible convection effect. The inner sphere is connected to a power regulator to maintain the input power constant during a single run. The power is turned on to heat the inner sphere and the data is collected when the inner and outer sphere temperatures remain almost constant. The processing time is about $4 \mathrm{~h}$. The same procedure is repeated for a new power setting for the same material.

\subsection{Thermal conductivity calculation}

For steady one-dimensional heat conduction in a spherical wall, neglecting convection and radiation, the heat equation reduces to:

$$
\mathrm{q}_{\mathrm{r}}=-\lambda \mathrm{A} \frac{\mathrm{d} T}{\mathrm{~d} r}
$$

For heat conduction through the wall held between two concentric spheres, the heat transfer can be expressed as:

$$
Q=\frac{t_{1}-t_{2}}{\frac{1}{2 \pi \lambda}\left(\frac{1}{d_{1}}-\frac{1}{d_{2}}\right)},
$$


where $t_{1}$ and $t_{2}$ are the inner sphere and the outer sphere temperatures, and $d_{1}$ and $d_{2}$ are the inner and outer diameters of the wall, respectively. The thermal conductivity coefficient can then be calculated at an average temperature of $\bar{t}=\left(t_{1}+t_{2}\right) / 2$,

$$
\lambda(\bar{t})=\frac{Q\left(d_{2}-d_{1}\right)}{2 \pi d_{1} d_{2}\left(t_{1}-t_{2}\right)} .
$$

\subsection{Experimental uncertainties}

The experimental uncertainty in the thermal conductivity coefficient in eqn (3) was estimated using [6],

$$
d \lambda=\left[\left(\frac{\partial \lambda}{\partial Q} \mathrm{~d} Q\right)^{2}+\left(\frac{\partial \lambda}{\partial t_{1}} \mathrm{~d} t_{1}\right)^{2}+\left(\frac{\partial \lambda}{\partial t_{2}} \mathrm{~d} t_{2}\right)^{2}\right]^{1 / 2}
$$

The temperature uncertainty was $0.1^{\circ} \mathrm{C}$, while the heat transfer uncertainty was $0.5 \%$. Thus, the uncertainty in thermal conductivity did not exceed $\%$. The effect of natural convection on the outside of the outer sphere was investigated using the commercial CFD software Fluent 6.0. The problem considered was a two-dimensional flow of air as a Boussinesq fluid and the temperature gradient on the sphere was less than $0.1^{\circ} \mathrm{C}$.

\subsection{Results and discussion}

A steady state method was used to measure the thermal conductivity of different natural, low-cost materials (coconut fibers and groundnut shell). A $70 \mathrm{~mm}$ thick sample was held between the two spherical enclosures. Data was collected when the temperatures of the inner and outer spheres were constant after about $4 \mathrm{~h}$ of heating. Both convection and radiation heat transfer in the insulation were assumed to be negligible. Measurements for different input powers gave results for temperature ranging from $27^{\circ} \mathrm{C}$ to $100^{\circ} \mathrm{C}$. The thermal conductivities of coconut fiber and the groundnut shell were determined by solving the heat conduction equation in spherical systems. The temperature dependence of the thermal conductivity was measured for both coconut fiber and groundnut shell. For the purpose of comparison, the same experimental procedure was done for s a standard and manmade insulation material such as aluminum silicate fiber. The results showed that the thermal conductivity of aluminum silicate fiber is lower than that of coconut fiber and groundnut shell. However, for all the three materials, the thermal conductivity coefficient increases with temperature (Fig. 4). The thermal conductivity of coconut fiber was initially less than that of the groundnut shell and became higher at about $80^{\circ} \mathrm{C}$ (Fig. 4). The thermal conductivity varies in the same manner for both coconut fiber and aluminum silicate fiber with less effect of temperature at temperatures less than $40^{\circ} \mathrm{C}$.

Actually, the systems composed of the solid material and air are characterized by an effective thermal conductivity which depends on the thermal conductivity and surface radiative properties of the solid material as well as the nature and volumetric fraction of the air or void space. The thermal conductivity also depends on the bulk density which in turn depends on the manner in which the solid material is interconnected. Thus, the heat is actually transferred by conduction through the 


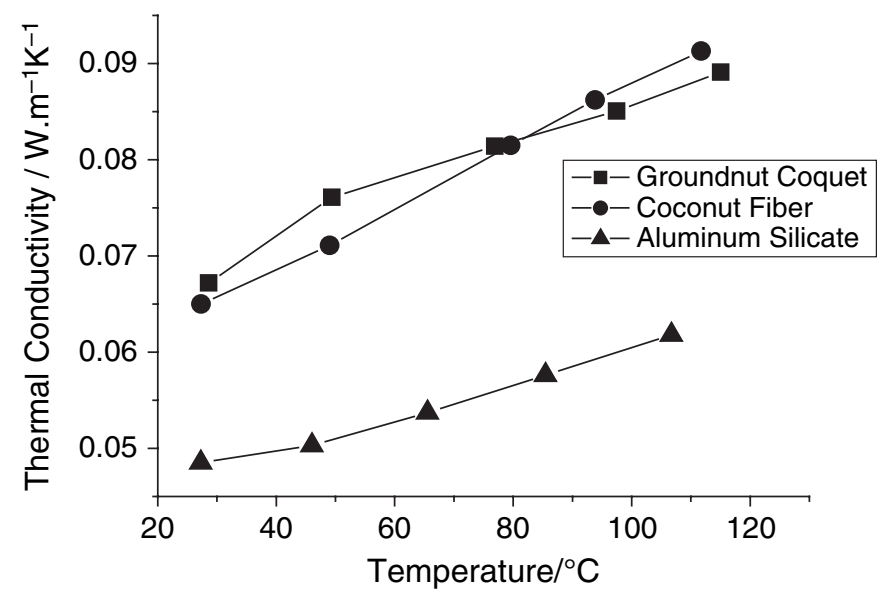

Figure 4: Temperature dependence of the thermal conductivity.

fibrous or porous solid matrix, conduction and convection of the air trapped in the void spaces, and, at sufficiently high temperatures, radiation.

\subsection{Test samples}

\section{EXPERIMENT FOR MOISTURE ABSORPTION}

The experimental samples for water absorption analysis were prepared as three rectangular boxes (30 mm thick and $100 \times 80 \mathrm{~mm}$ ) made with wire mesh (wire diameter $0.071 \mathrm{~cm}, 315$ strands per meter) and then filled with the dried materials to be tested. The experimental samples $(90 \mathrm{~mm}$ thick and $100 \times 80 \mathrm{~mm}$ dimensions) were sandwich multi-layers composed of three rectangular layers filled with the test materials - coconut fibers or groundnut shell. The test materials should be carefully packed to achieve the greatest density and reduce the amount of air. However, it was very difficult to obtain high material density for the coconut fiber sample due to the structure of the material. So, the sample made with coconut fiber had higher void fraction than the groundnut shell sample.

\subsection{Test facility}

The experimental setup to analyze the moisture absorption by the test materials is shown in Fig. 5 . Polyester was used to make an enclosure with inner dimensions of $400 \times 300 \times 300 \mathrm{~mm}$ and a $90 \mathrm{~mm}$ thick plate which has a rectangular gap of $100 \times 80 \mathrm{~mm}$ in the center, where the sandwich multilayer sample will be placed. The bottom wall of the enclosure has a gap of $100 \times 100 \mathrm{~mm}$ for vapor recirculation, and there is another gap in the top wall to put and remove the samples. A wire mesh is used to cover the samples. An electric boiler is used to produce water vapor at about $100^{\circ} \mathrm{C}$. A metal plate $(300 \times 300 \mathrm{~mm})$ is used to support the samples. The plate has several little holes at the center where the testing materials will be placed so that the vapor can flow through the sample from the bottom layer to the top surface (Fig. 5). An impermeable plastic sheet is used to isolate the samples from the ice and to prevent the melted ice (or water) from flowing downwards onto the samples. 


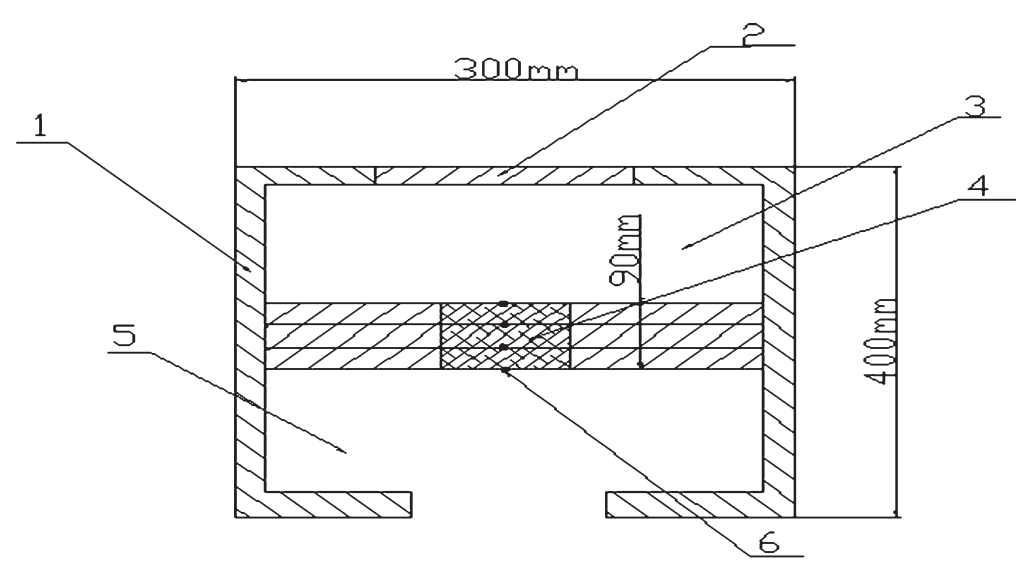

Figure 5: Experimental apparatus for water absorption analysis. 1 - Insulated box; 2 - gap window; 3 - space for ice; 4 - layers of test materials; 5 - vapor recirculation; 6 - thermocouple connections.

An electronic balance is used to weigh the samples before and after testing. A multi-meter is used for the thermocouple connections to control the temperatures.

\subsection{Experimental procedure}

The experimental procedure is as follows. The samples are weighed using an electronic balance and then the sample layers are placed on the plate through the gap in the $90 \mathrm{~mm}$ thick wall at the center of the enclosure. The thermocouples are connected to the horizontal surfaces of the layers and the temperature of each layer is measured before testing. A plastic sheet is placed on the top surface of the top layer. Ice cubes are placed in the upper compartment of the enclosure and water vapor is supplied to the compartment below so that the test materials are bound by ice at the top and the bottom surface is exposed to vapor. After $1 \mathrm{~h}$ of testing, the samples are removed and weighed immediately. Then the water content is calculated using eqn (5). The same procedure is repeated for $t=2 \mathrm{~h}, 3 \mathrm{~h}, 4 \mathrm{~h}$.

$$
w_{\mathrm{ac}}=\frac{w_{\mathrm{i}}-w_{\mathrm{o}}}{w_{\mathrm{o}}} 100 \%
$$

where $w_{\mathrm{i}}$ and $w_{\mathrm{o}}$ are the weights of the layer after testing and before testing, respectively, and $w_{\text {ac }}$ is the increase in water content as a percentage. The results are shown in Fig. 6; $X$ is the dimensionless distance from the layer one side of which is exposed to vapor.

$$
X=\frac{x_{\mathrm{i}}}{d}
$$

where $x_{\mathrm{i}}$ is distance from the surface exposed to vapor $\left(x_{\mathrm{i}}=0\right.$ at the bottom and $x_{\mathrm{i}}=90 \mathrm{~mm}$ at the top) and $d$ is the total thickness of the sample. 
The experimental uncertainty in weight is $0.1 \mathrm{~g}$; and thus, according to the procedure in [6], the uncertainty is about $6 \%$ in water content.

\subsection{Results and discussion}

\subsubsection{General behavior}

A sandwich multi-layer of the test materials was bounded at the top by ice and the bottom surface was exposed to vapor with temperature close to $100^{\circ} \mathrm{C}$. Before testing, the temperature at each location of the thermocouples on the sample was about $20^{\circ} \mathrm{C}$. After $1 \mathrm{~h}$ of testing, the temperature at the bottom reached a maximum of $98^{\circ} \mathrm{C}$ and remained almost unchanged. At the top surface, which was cooled by ice, the temperature first decreased slightly and then increased to about $30^{\circ} \mathrm{C}$ after $1 \mathrm{~h}$ and finally reached $35^{\circ} \mathrm{C}$ after $4 \mathrm{~h}$. The percentage of water accumulation due to vapor absorption was calculated for each layer. Figure 6 presents the measurements of water absorption as a function of the non-dimensional distance. The water content within the coconut fiber and groundnut shell increased with time, and the water content for the groundnut shell was higher than that of the coconut fiber. By comparing the results for the two samples, one can easily see the differences in the water content distributions and their variation with time. The water accumulation and distribution within the test material were due to the transport of warm and moist air through the material toward the cold surface. At some location within the insulation in the cold region, the insulation temperature would be below the saturation temperature and moisture would condense and soak the material. The water content distribution is also affected by the liquid motion on the fiber surfaces. When the amount of liquid condensate reaches a certain threshold, the liquid water overcomes the surface tension effects and moves to regions with lower water content. These effect are discussed in the following sections with numerical simulation.

3.4.2 Effect of the pore scale and specific surface area

For the groundnut shell sample having small pore scale and high specific surface area, the water content was significantly higher than that of the coconut fiber, and also the increase in water content was very fast and significant for the layer exposed to water vapor within $2 \mathrm{~h}$; however, the increase

(a)

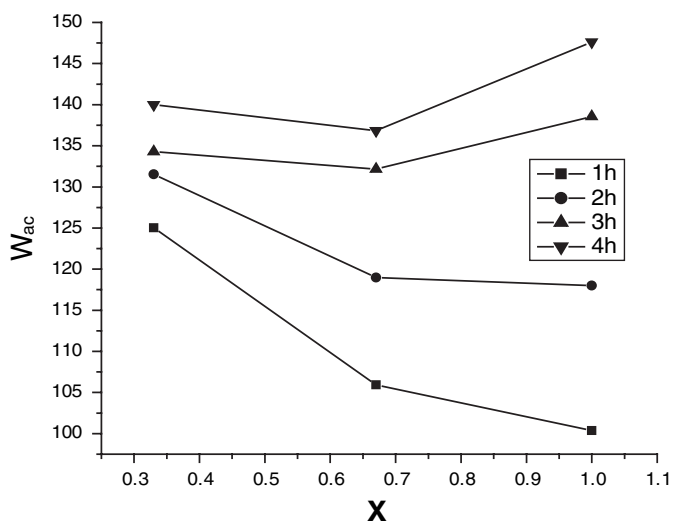

(b)

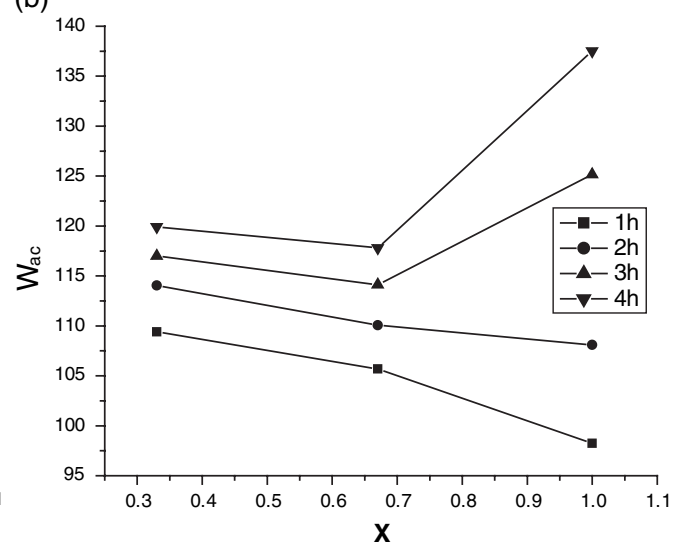

Figure 6: Water content $\left(w_{\mathrm{ac}}\right)$ versus non-dimensional distance $(X)$ : (a) groundnut shells; (b) coconut fibers. 
in the top layer water content was faster for the coconut fiber sample (Fig. 6b). For the groundnut shell sample (Fig. 6a), the water vapor first condensed within the bottom layer and increased its water content before flowing through the middle layer toward the top layer, where the condensation occurred further due to the much lower temperature compared with that in the bottom layers. Therefore, the water content in the top layer finally increased due to the condensation and the impermeable cold surface top which did not allow vapor and condensed water to flow through the sample. A part of the condensed water from the top layer flowed back into the middle layer owing to gravity, and from the bottom layer to the middle region driven by the capillary force (Fig. 6a). Thus, two motions of fluid were observed: vapor flow from the bottom layer toward the middle and top layers and the condensate water flow from the top and bottom layers to the middle layer. Similar phenomena and processes occurred for the coconut fiber sample. However, the coconut fiber samples had high pore scale which allowed the vapor to flow easily through the samples toward the impermeable top surface, and the much faster increase in water content in the top layer than in the groundnut shell sample was induced due to the intensity condensation and water accumulation. The condensation mainly occurred in the top layer of the coconut fiber sample. Also, the first two layers (from the surface exposed to vapor) were wet almost uniformly during the entire experiment with higher water content for the first layer (Fig. 6b).

\subsubsection{Influence of effective thermal conductivity}

Many available investigations indicate that the fluids and their behavior, existing in the pores of porous materials, could significantly alter the energy transport behavior of these materials. Normally, this effect is considered as effective thermal conductivity including the contribution of solid matter, liquid, and gas, and even radiation [7]. Particularly, water content, generally described using a parameter of water phase saturation or $S$ (void volumetric fraction occupied by a liquid) would apparently increase the effective thermal conductivity, as water is a better thermal medium than usual insulation materials.

The following equation of thermal conductivity assumes that the liquid is distributed in continuous layers parallel to the direction of heat flow [7].

$$
\lambda_{\text {eff }}=(1-\varepsilon) \lambda_{\mathrm{s}}+\varepsilon\left(S_{1} \lambda_{1}+S_{\mathrm{g}} \lambda_{\mathrm{g}}\right)
$$

where $\varepsilon$ is the porosity; $\lambda_{\mathrm{s}}, \lambda_{\mathrm{l}}$, and $\lambda_{\mathrm{g}}$ are the thermal conductivities of solid matter, liquid, and gas, respectively; $S_{1}$ and $S_{\mathrm{g}}$ are the phase saturation for liquid and gas (volumetric fraction of the void space occupied by a specific phase).

Equation (7) clearly shows that the effective thermal conductivity of moist insulations depends considerably on the amount of the liquid contained, or increases with increasing water content. For practical applications of natural materials as insulators, their water absorption, and inner transport phenomena, should be one of the important aspects that would destroy the insulating capacity of these materials. Consequently, some efficient ways should be considered for decreasing water absorption.

\section{SIMULATION OF MASS TRANSPORT}

\subsection{Formulation and numerical methods}

For a simple theoretical understanding of the experimental observation, especially the water accumulation process, consider a one-dimensional unsteady problem of heat and mass transfer with 


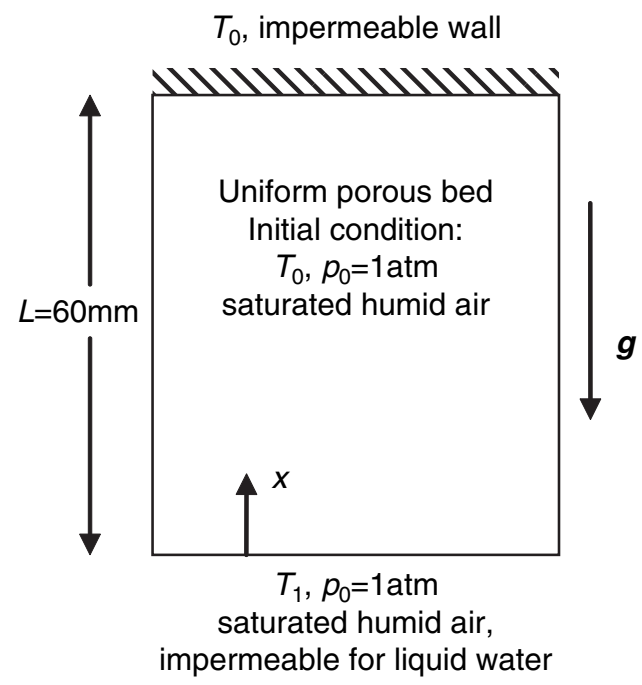

Figure 7: Problem depiction.

condensation in a porous medium with a porosity of 0.36 (Fig. 7), which is composed of spherical particles with a thermal conductivity of $1 \mathrm{~W} /(\mathrm{m} \mathrm{K})$ and diameter of $0.1 \mathrm{~mm}$. On the top of the porous bed there is a cold impermeable wall, and the bottom of the bed is waterproof and only permeable for the gas phase. Initially, the porous bed is filled with saturated humid air. The bottom is exposed to hot saturated humid air, while the top wall temperature is maintained constant. In Fig. 7, the temperatures $T_{\mathrm{o}}=30^{\circ} \mathrm{C}$ and $T_{1}=70^{\circ} \mathrm{C}$.

The calculation was carried out based on the following mechanisms:

1. Water vapor mass conservation, including convection, diffusion, and phase change.

2. Air mass conservation, including convection and diffusion.

3. Liquid water conservation, including phase change and convection by gravity and capillary pressure [8-10].

4. Momentum transfer obeying Darcy's law [11].

5. Heat transfer by convection, diffusion, and latent heat release by phase change.

6. Variable thermal properties, including the influence of water accumulation on effective diffusivity and thermal conductivity.

\subsection{Numerical results}

The transfer process can be divided into two distinct stages, the early stage and the late stage, characterized by their dominant mechanisms. During the early stage, when $t<2000 \mathrm{~s}$, the temperature field is far from the steady distribution and the rapid change of the temperature field results in a remarkable change in the condensate rate, as shown in Fig. 8. First, vapor condenses mostly near the bottom of the porous bed, and then the condensation rate $\dot{m}_{\mathrm{w}}$ near the bottom decreases, but the condensation area extends to the upper part. After $400 \mathrm{~s}, \dot{m}_{\mathrm{w}}$ at the top increases significantly and it gradually exceeds that in other positions. The irreducible water saturation $S_{\text {ir }}$, below which water cannot form a continuous film or flow in porous media, is taken as $0.09[9,10]$. Since the water saturation $S$ is lower than the irreducible saturation $S_{\text {ir }}$ in this early stage, the condensate cannot form a continuous liquid film to flow. $S$ is proportional to the integral of $\dot{m}_{\mathrm{w}}$ on time, decreasing from the 
(a)

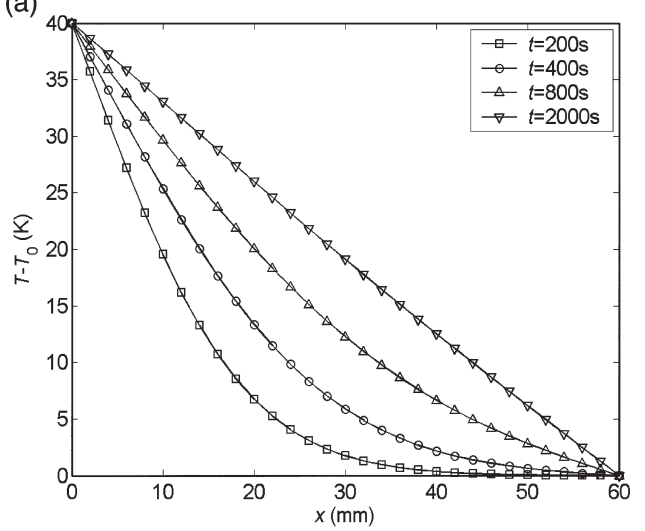

(b)

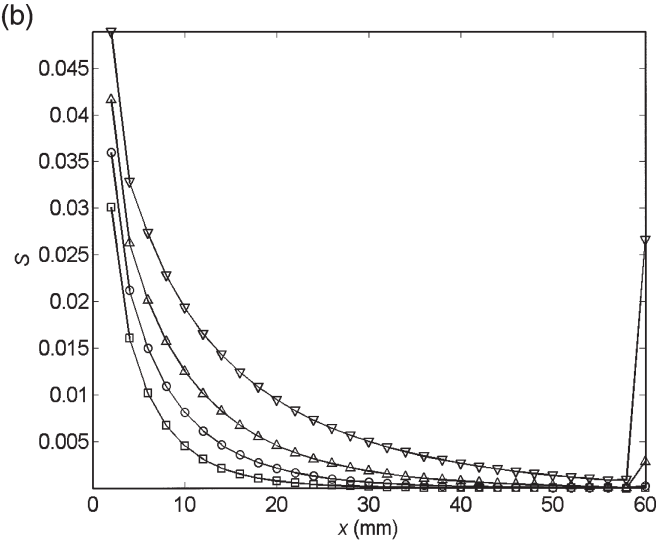

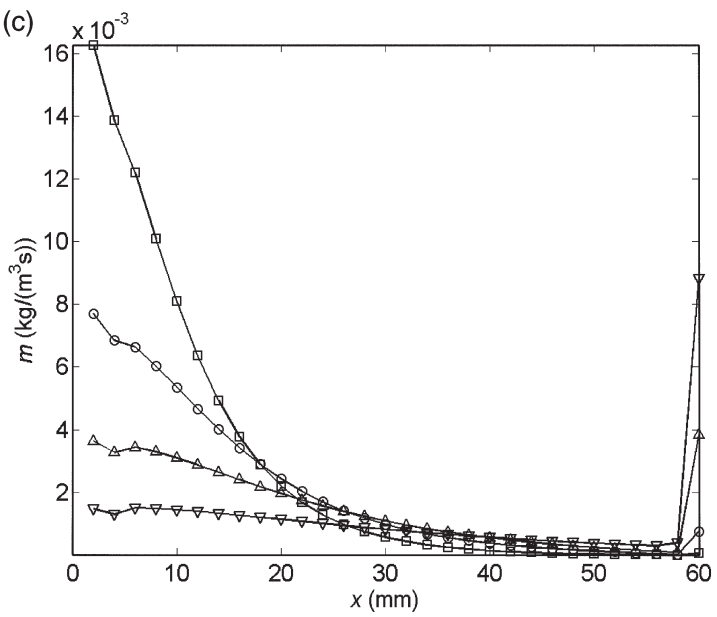

Figure 8: Distributions in the early stage: (a) temperature difference; (b) liquid water saturation; (c) condensation rate.

bottom to the top, but at the top, there is a jump because of high $\dot{m}_{\mathrm{w}}$. The condensation rate achieves a very high value at the top wall (Fig. 9c), due to the impermeable boundary and the lowest temperature. The peaks in temperature gradient are caused by the rapid variation of the effective thermal conductivity, which is caused by the rapid change in water content. The peaks can be observed in the positions where the water saturation changes rapidly in Fig. 9b. This is also the reason for the peaks in condensation rate in Fig. 9c. These characteristics match with the experimental observations shown in Fig. 6.

During the late stage, when $t>2000 \mathrm{~s}$, temperature profiles at different times overlap, and the temperature gradient grad $T$ is shown to depict the slow change of the temperature field in detail (Fig. 9). In this figure, the negative liquid water velocity $v_{\mathrm{w}}$ denotes downward flow, and positive $v_{\mathrm{w}}$ denotes upward flow. Obviously, liquid water is sucked from both the top and the bottom to the middle, because $\dot{m}_{\mathrm{w}}$ and $S$ on both sides are higher than that in the middle. When $S$ reaches $S_{\mathrm{r}}, S$, grad $T$, and $\dot{m}_{\mathrm{w}}$ all rapidly change spatially. Thus, the results in Fig. 6 support the results obtained from the numerical simulation. As time proceeds, $S$ increases slowly and thermal properties, including the effective diffusivity $D_{\text {eff }}$ and effective thermal conductivity $\lambda_{\text {eff }}$, change, which causes the redistribution of other variables. 
(a)

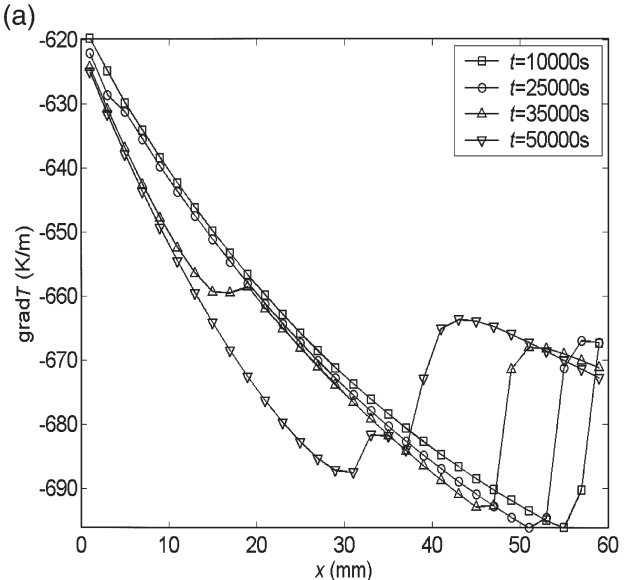

(c)

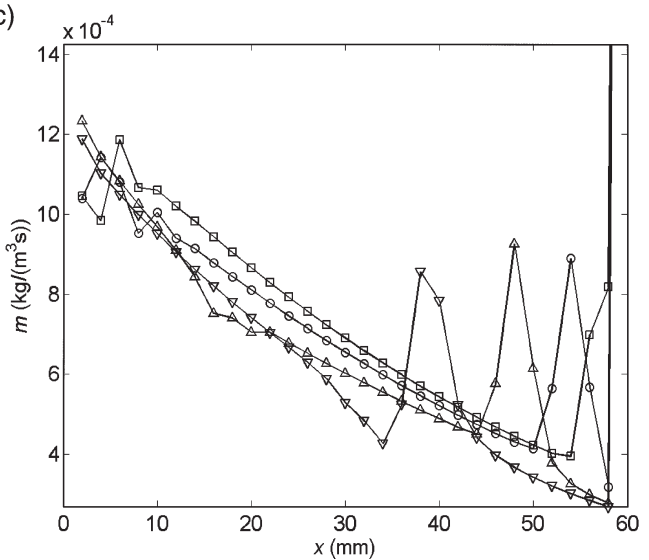

(b)

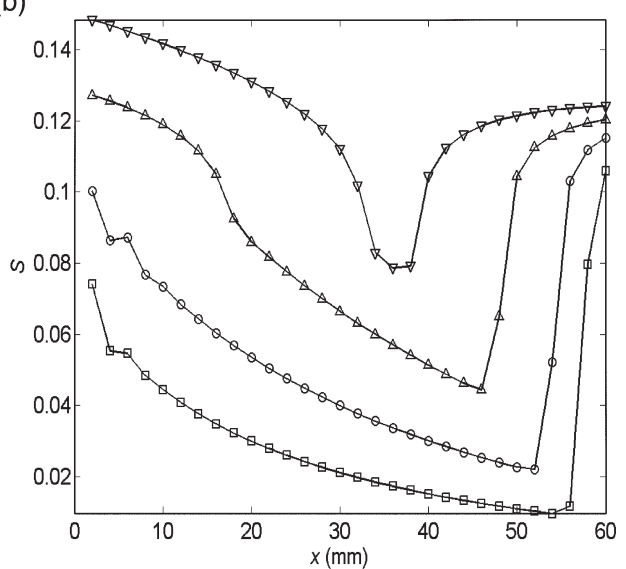

(d)

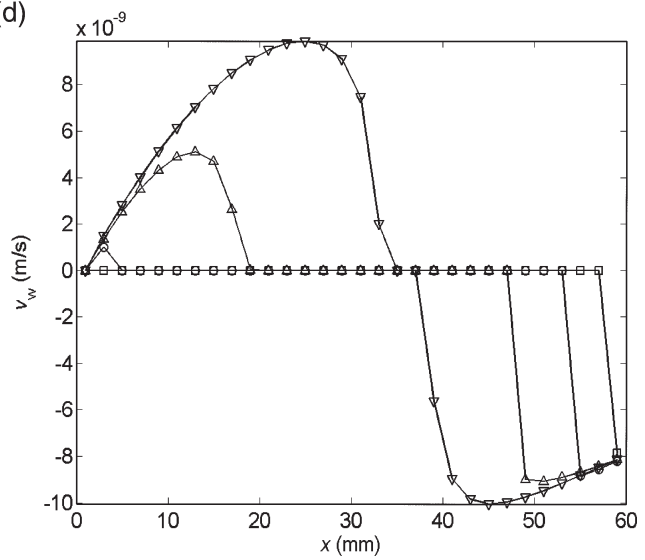

Figure 9: Distributions in late stage: (a) temperature gradient; (b) liquid water saturation; (c) condensation rate; (d) liquid water velocity.

To summarize, the early stage is characterized by the rapid change of $T$ and the corresponding redistributions of mass and $\dot{m}_{\mathrm{w}}$, while the late stage is characterized by the slow accumulation of $S$, corresponding to a slow change of thermal properties and slow redistributions of $v_{\mathrm{w}}$ and $\dot{m}_{\mathrm{w}}$. These are in good agreement with some reported results [12]. The trend of the $S$ profile was in accordance with both numerical and experimental investigations conducted by Larbi et al. (under condition D) using the de Vries model [13]. In that study, the simulation model used is based on the volume-averaging method.

\section{CONCLUDING REMARKS}

The transport properties of two natural low cost materials - coconut fibers and groundnut shell - were investigated experimentally. The materials were placed between two concentric spheres for the tests. Heat was transferred mainly by conduction through the materials. The results showed that the thermal conductivities of both coconut fiber and groundnut shell sample increase with temperature in the same manner as the thermal conductivity of the standard insulating material (aluminum silicate fibers). Also, experimental investigations were done to characterize the moisture absorption of two different 
natural materials (coconut fibers and groundnut shell). A sandwich multi-layer $(100 \times 80 \times 30 \mathrm{~mm}$ dimensions of each layer) of the test materials was bounded on top by ice and the bottom surface was exposed to vapor with temperature close to $100^{\circ} \mathrm{C}$. The percentage of water accumulation due to vapor absorption was calculated for each layer after a pre-set time of experiment $(1 \mathrm{~h}, 2 \mathrm{~h}, \ldots)$.

The mass and heat transfer was simulated by considering the coupling of the heat transfer, mass transfer, phase change, and variable physical properties. Both experimental and simulation results showed that the water vapor first condensed within the bottom layer and increased its water content before flowing through the middle layer toward the top layer where condensation occurred further due to the low temperature. Part of the condensed water from the top layer flowed back to the middle layer because of gravity, and from the bottom layer to the middle region driven by the capillary force. Two motions of fluid were observed: vapor flowing from the bottom layer toward the middle and top layers, and the condensate water flowing from the top and bottom layers to the middle layer driven by capillary force and gravity, respectively. A simple numerical simulation was also conducted for the water content migration behavior in a porous medium to theoretically understand the experimental observations. The results demonstrated good agreement with the mass transport behavior shown in Fig. 6.

The measured thermal conductivities showed that coconut fibers and groundnut shells may be used as thermal insulators in the tested temperature range or lower. However, the thermal conductivity of moist insulation depends considerably on the amount of the liquid it contains; as a result, since water is a better thermal medium, the thermal conductivity of the soaked insulation would increase and the insulating capacity of the material would be destroyed. A thin vapor barrier should be placed over the insulation to prevent moisture from entering the insulation.

Actually, although the thermal conductivity is the most important thermal insulation parameter, there are also other important criteria such as the environment (raw renewable materials, ozone depletion, energy consumption, waste processing, etc.) and health (allergies, turbid respiration, cancers, etc.) to be considered for selecting good thermal insulation. Some natural materials such as coconut fiber and groundnut shell are the best insulation materials owing to their harmlessness to the environment and their unlimited availability in nature.

\section{ACKNOWLEDGEMENTS}

The authors gratefully acknowledge the financial support of the National Natural Science Foundation of China (No.50636030).

\section{NOMENCLATURE}

$d$

$g \quad$ acceleration due to gravity $\left(\mathrm{m}^{2} / \mathrm{s}\right)$

$\operatorname{grad} T \quad$ temperature gradient $(\mathrm{K} / \mathrm{m})$

$m \quad$ condensation rate $\left(\mathrm{kg} / \mathrm{m}^{3} \mathrm{~s}\right)$

$S \quad$ phase saturation

$t \quad$ temperature (K) in eqns (2-4); time (s) in Figs 8-9

$T \quad$ temperature $(\mathrm{K})$

$V_{w} \quad$ velocity $(\mathrm{m} / \mathrm{s})$

$X^{w} \quad$ non-dimensional distance

$\lambda \quad$ thermal conductivity $(\mathrm{W} / \mathrm{m} \mathrm{K})$

$\varepsilon \quad$ porosity 


\section{REFERENCES}

[1] Papadopoulos, A.M., State of the art in thermal insulation materials and aims for future developments. Energy and Buildings, 37, pp. 77-86, 2005.

[2] Koelet, P.C. \& Gray, T.B., Industrial Refrigeration: Principles, Design and Applications, Macmillan: London, pp. 234-384, 1992.

[3] Kodah, Z.H., Jarrah, M.A. \& Shanshal, N.S., Thermal characterization of foam-cane (Quseab) as an insulant material. Energy Conservation and Management, 40, pp. 349-367, 1998.

[4] Fan, J., Cheng, X. \& Chen, Y.-S. An experimental investigation of moisture absorption and condensation in fibrous insulations under low temperature. Experimental Thermal and Fluid Science, 27, pp. 723-729, 2002.

[5] Edwin, F.S. \& William, C.T., Thermal Insulation Building Guide, Robert E. Krieger Publishing Company: Malabar, pp. 1-473, 1990.

[6] Holman, J.P. \& Gajda, W.J., Experimental Methods for Engineers, 5th edn. McGraw-Hill: New York, pp. 37-83, 349-379, 1989.

[7] Choudhary, M.K., Karki, K.C. \& Patankar, S.V., Mathematical modeling of heat transfer, condensation, and capillary flow in porous insulation on a cold pipe. International Journal of Heat and Mass Transfer, 47, pp. 5629-5638, 2004.

[8] Ergun, S., Fluid flow through packed columns. Chem. Eng. Proc., 48, pp. 89-94, 1952.

[9] Udell, K.S., Heat transfer in porous media heated from above with evaporation, condensation, and capillary effects. Journal of Heat Transfer, 105, pp. 485-492, 1983.

[10] Udell, K.S., Heat transfer in porous media considering phase change and capillarity - the heat pipe effect. International Journal of Heat and Mass Transfer, 28, pp. 485-495, 1985.

[11] Darcy, H., Les Fontaines Publiques de la ville de Dijon, Dalment: Paris, 1856.

[12] Wijeysundera, N.E., Zheng, B.F., Iqbal, M., \& Hauptmann, E.G., Numerical simulation of the transient moisture transfer through porous insulation. International Journal of Heat and Mass Transfer, 39, pp. 995-1004, 1996.

[13] Larbi, S., Bacon, G., \& Bories, S.A., Humid air diffusion with water vapor condensation in porous media. International Journal of Heat and Mass Transfer, 38, pp. 2411-2426, 1995. 